\title{
Anabases
}

ANABASES Traditions et réceptions de l'Antiquité

$9 \mid 2009$

Varia

\section{Introduction : Alésia et la mémoire nationale française}

Michel Reddé

\section{(2) OpenEdition}

1 Journals

Édition électronique

URL : http://journals.openedition.org/anabases/99

DOI : 10.4000/anabases.99

ISSN : 2256-9421

Éditeur

E.R.A.S.M.E.

Édition imprimée

Date de publication : 1 mars 2009

Pagination : 13-24

ISSN : 1774-4296

\section{Référence électronique}

Michel Reddé, «Introduction : Alésia et la mémoire nationale française », Anabases [En ligne], 9| 2009, mis en ligne le 01 mars 2012, consulté le 10 décembre 2020. URL : http://journals.openedition.org/ anabases/99; DOI : https://doi.org/10.4000/anabases.99 
Anabases 9 (2009), p. 13-24.

\section{Introduction :}

\section{Alésia et la mémoire nationale française}

Michel RedDÉ

Dans l'introduction à son ouvrage sur l'Histoire de Jules César, publié en $1866^{1}$, Napoléon III justifiait son entreprise d'érudition par une leçon de politique: « Ce qui précède, écrivait-il, montre assez le but que je me propose en écrivant cette histoire. Ce but est de prouver que, lorsque la Providence suscite des hommes tels que César, Charlemagne, Napoléon, c'est pour tracer aux hommes la voie qu'ils doivent suivre, marquer du sceau de leur génie une ère nouvelle et accomplir en quelques années le travail de plusieurs siècles. Heureux les peuples qui les comprennent et les suivent. Malheur à ceux qui les méconnaissent et les combattent. " De manière implicite mais évidente, l'Empereur se situait lui-même dans cette lignée, et cette autoglorification, doublée de la justification d'un régime autoritaire qui s'est écroulé à Sedan, n’a pas peu contribué à jeter le discrédit sur une entreprise archéologique considérable, parfois soupçonnée de "forgerie ", notamment dans le cas d'Alésia. D'assez nombreux travaux universitaires ont, depuis plusieurs années, remis à leur juste place les recherches du Second Empire en les situant dans un vaste mouvement européen ${ }^{2}$. C'est l'objectif de cette journée d'étude que d'en préciser quelques aspects nouveaux et d'examiner quelle place l'archéologie a tenu dans la conservation, voire la fabrication, de la mémoire nationale, en France et en Italie. Pour ma part, et compte tenu du sujet, j'y ajouterai volontiers quelques considérations sur l'Allemagne.

1 (Anonyme), Histoire de Jules César, Paris, 1865-1866. Il existe trois éditions de formats différents $\left(\mathrm{F}^{\circ}, 4^{\circ}, 8^{\circ}\right)$.

2 Il n'est pas possible d'en donner ici une bibliographie exhaustive, qui serait considérable. Nous citerons à mesure les travaux les plus importants pour notre propos. 
Dans La conquête du passé, A. Schnapp a analysé de manière détaillée le lent processus intellectuel qui a conduit à l'invention d'une science archéologique. Progressivement détachée de la philologie, elle se distingue peu à peu de l'approche traditionnelle des Antiquaires en intégrant dans sa réflexion l'apport des sciences naturalistes, inventant ainsi, sous l'influence des préhistoriens, la notion de " $\operatorname{contexte}^{3}$ ". Le temps de la seule jouissance esthétique que procurait l'Art antique faisait ainsi place à une approche " positive " des objets, même les plus humbles ou les plus communs, et à une investigation raisonnée du terrain. Cette évolution épistémologique a marqué toute l'Europe, naturellement de manière différente et avec plus ou moins de rapidité selon les pays: les préoccupations urbanistiques de la Rome papale, confrontée quotidiennement aux monuments de l'Antiquité, présents depuis toujours au cœur de la Ville, n'étaient pas de même espèce et n'avaient pas les mêmes implications que la recherche sur les tumuli protohistoriques; nous savons bien, à l'aube du XXI siècle, que cette lente évolution de l'archéologie en "science de la nature " n'est toujours pas achevée, qu'elle rencontre encore de fortes résistances, qu'elle est toujours fortement inégale dans la pratique quotidienne, d'un pays à l'autre.

Dans ce processus de maturation de la discipline, on doit distinguer plusieurs étapes. C'est en pleine période romantique, et malgré la concurrence qui sévissait alors entre les États pour l'acquisition d'Antiquités grecques et romaines, que les savants de toute l'Europe s'organisèrent spontanément, créant en 1829, à la légation de Prusse, un Istituto di corrispondenza archeologica. Celui-ci a constitué de fait, bien avant l'Unité italienne, un "ministère officieux ${ }^{4}$ ", qui n'allait pas survivre longtemps à la montée des rivalités nationales.

Dans l'Allemagne de ce temps, l'érudition était d'abord le fait des Universités, où l'archéologie était enseignée, mais il n'en allait pas de même en France, où le système universitaire était (déjà) en retard. Cette époque fut en revanche marquée, dans notre pays, par le foisonnement des sociétés savantes, dont l'activité multiforme touchait la province autant que Paris. Partout on cherche, on discute, on publie, en un temps où tout notable qui a fait ses humanités se pique de science et d'écriture. Période bouillonnante et féconde, parfois brouillonne, pendant laquelle on produit beaucoup, mais qui compte aussi ses personnages ridicules, comme ces "Bouvard et Pécuchet ", moqués par G. Flaubert dans un de ses romans les plus féroces. Dans cet État pourtant centralisé depuis longtemps, le retard de l'action publique est patent, les moyens financiers limités si on les compare à ceux du grand pays voisin en train de se rassembler. Le mécénat privé n'y tient pas la même place essentielle qu'en Angleterre, où les sociétés savantes jouait là aussi un rôle primordial. C'est pour pallier ces carences que Guizot créa, en 1834, le Comité des Travaux Historiques, dont la vocation était de coordonévidemment pas encore employé au XIX ${ }^{\mathrm{e}}$ siècle. 
ner l'action des sociétés, de financer leurs recherches par des subventions ouvertes au budget de l'État à partir de 1847. Cette action de pilotage du CTHS a été fondamentale, notamment après la conquête de l'Afrique du Nord, mais elle a parfois été considérée comme une mise sous tutelle par les intéressés 5 .

Parallèlement, l'archéologie commençait à servir une quête identitaire d'une nouvelle espèce. Ce n'est plus seulement l'histoire de l'Humanité dans son caractère universel que l'on voulait écrire, comme au XVIII ${ }^{\mathrm{e}}$ siècle, mais celle des Nations. Dans cette "guerre culturelle qui se déroulait en contrepoint de la politique européenne ", pour reprendre une expression de $\mathrm{Cl}$. Nicolet ${ }^{6}$, l'un des principaux enjeux était de capter l'héritage de la Rome antique et d'instrumentaliser l'histoire romaine à son profit ${ }^{7}$. Alors que F. Schiller pouvait encore écrire, en 1797: «Deutschland? Aber wo liegt es? Ich weiss das Land nicht zu finden ", tout change après les guerres napoléoniennes. La recherche de l'emplacement de la bataille du Teutoburg ne relève plus seulement d'un problème académique mais d'une approche " politique » où le lieu - nécessairement une forêt sert de fondement symbolique à la mythologie germanique et à la défense du " Deutsches Volk» contre les invasions venues de l'Ouest ${ }^{8}$. Si on ne parle pas encore d'État à cette époque en Allemagne, le parallèle entre le Varusschlacht et la "Bataille des Nations ", comme on l'appelle alors (id est la bataille de Leipzig), est dans tous les esprits. Mais, dans le même temps, l'Allemagne se présente comme la meilleure héritière des valeurs de la Grèce et de Rome; bientôt elle en revendiquera de nouveau l'héritage politique, renouant ainsi avec la tradition du Saint Empire. En France, comme l'a bien montré Chr. Goudineau, ce sont les frères Thierry, Amédée en particulier, qui ont lancé l'idée d'une France enracinée dans ses origines gauloises. L' Histoire des Gaulois depuis les temps les plus reculés jusqu'à l'entière soumission de la Gaule à la domination romaine paraît en 1828; texte fondateur qui introduit véritablement Vercingétorix comme personnage historique. Quelques années plus tard (1833) Henri Martin se lance à son tour dans une Histoire de France qui va populariser, avec un immense succès, la série des Héros qui ont fait notre

5 Sur toute cette période et sur l'organisation de l'archéologie française en général, voir E. Gran-Aymerich, Naissance de l'archéologie moderne. 1798-1945, Paris, CNRs Éditions, 1998 ; sur Guizot, L. Theis, "Guizot et les institutions de mémoire", dans P. Nora (éd.), Les lieux de mémoire, I, Paris, $1997^{2}$, p. 15-75.

$6 \mathrm{Cl}$. Nicolet, "Napoléon III et l'histoire romaine", Introduction à l'ouvrage de M.A. Tomei, Scavi francesi sul Palatino. Le indagini di Pietro Rosa per Napoleone III (1861-1870), Rome, 1999, p. IX-XXI.

7 L'étude en a été faite, pour la France, par Cl. Nicolet, La fabrique d'une nation. La France entre Rome et les Germains, Paris, 2003.

8 Voir l'analyse d'A. Schnapp, “Teutoburger Wald et Alésia: deux figures de l'identité historique”, dans M. Reddé et S. von Schnurbein (éd.), Alésia et la bataille du Teutoburg. Un parallèle critique des sources, Beiheft der Francia, 66, 2008, p. 11-26, et celle de R. Wiegels, “'Varusschlacht' und 'Hermann' Mythos. Historie und Historisierung eines römisch-germanischen Kampfes im Gedächtnis der Zeiten”, ibid., p. 27-51. 
pays et dont Vercingétorix est le premier exemple ${ }^{9}$. Cette irruption des Gaulois dans la mémoire nationale se traduit par un nombre infini de productions littéraires (romans, poèmes, pièces, opéras), de libelles historiques, de représentations figurées plus ou moins fantaisistes dans les journaux, les arts mineurs, mais aussi la sculpture ou la grande peinture. Depuis les Trois Glorieuses, le Gaulois est à la mode. Contentons-nous, ici, de citer le tableau de Théodore Chassériau, au musée des Beaux-Arts de Clermont-Ferrand, $\mathrm{La}$ défense des Gaules, où l'on voit le fils de Celtil, dans une quasi nudité hérö̈que, exhorter les Gaulois à la bataille, les bras et les yeux levés au ciel (fig. 1).

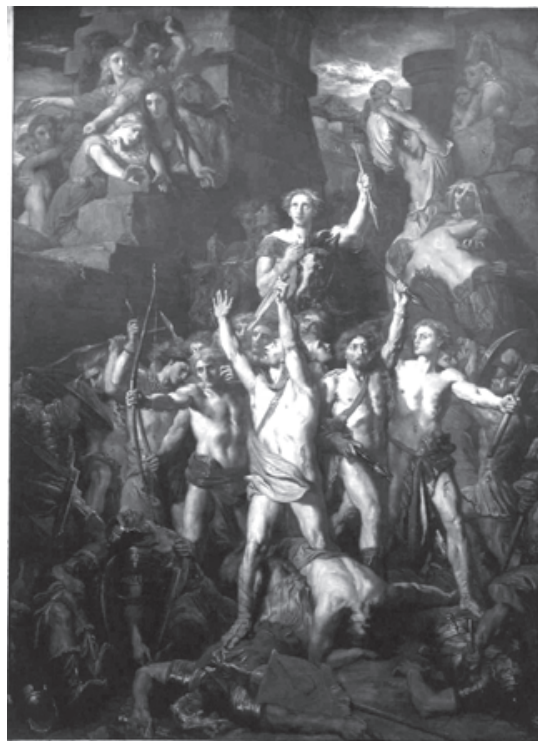

Fig. 1. La défense des Gaules (1855). tableau de Théodore Chassériau, collection du musée d'art Roger-Quillot, ville de Clermont-Ferrand

Ce tableau, qui reçut un accueil flatteur, fut exposé en 1855, une date qui n'est pas dénuée d'intérêt. C'est en effet cette même année que commence la "querelle d'Alésia ", qui va encombrer la presse française d'une invraisemblable série d'articles, d'opuscules plus ou moins savants, de pamphlets plus ou moins violents ${ }^{10}$. La question au cœur de ce débat n'est pas seulement académique, elle touche désormais à l'identité même de la Nation et à un mythe fondateur de l'histoire de France, devenu populaire en quelques années, qui remplace, pour les Républicains notamment, le baptême de Clovis, fondement de la monarchie. Les fouilles d'Alise-Sainte-Reine, remarquons-le, ne débuteront que bien plus tard, en 1861. La querelle les précède largement, elle n'est alors, en aucune manière, une contestation des recherches au pied du Mont-Auxois qui n'ont pas commencé.

On a donc grand tort de moquer la passion archéologique de Napoléon III : celle-ci n'est pas le caprice fantasque d'un tyran, elle est le fruit d'une époque, le produit d'une curiosité scientifique qui touche toute l'Europe, sur fond de rivalités nationales. Il n'est pas jusqu'à la célèbre statue de A. Millet, érigée en 1865 sur l'oppidum du Mont-Auxois (fig. 3), qui ne soit une réplique à la Prusse, dont on savait, depuis bien longtemps, qu'elle préparait un monument à Arminius (fig. 2). Certes la statue de Detmold ne fut inaugurée qu'en 1875, dans une Allemagne

9 Chr. Goudineau, Le dossier Vercingétorix, Paris, 2001; id. "La Gaule, les Gaulois, Vercingétorix et le sentiment national au XIX ${ }^{\mathrm{e}}$ siècle”, dans M. Reddé et $\mathrm{S}$. von Schnurbein (éd.), Alésia et la bataille du Teutoburg... (op. cit., note 8), p. 53-71.

10 Voir M. Reddé, Alésia. L'archéologie face à l'imaginaire, Paris, 2003; M. Reddé, "La querelle d'Alésia, hier et aujourd'hui”, dans M. Reddé et S. von Schnurbein (ed.), Alésia et la bataille du Teutoburg... (op. cit., note 8), p. 153-163. 
désormais unifiée, et sa base figure un relief de Guillaume $\mathrm{I}^{\text {er }}$, fondu dans le bronze des canons pris à Gravelotte; mais, dès 1843, le Magasin pittoresque se faisait l'écho du projet de statue, confié au sculpteur E. von Bandel:

Les Allemands élèvent aujourd'hui dans la forêt de Teutoburg, témoin de la défaite de Varus, une statue colossale à Arminius, qu'ils nomment Hermann... Ils entendent qu'elle représente l'hostilité de leur race contre les races qui ont hérité en Europe de la puissance ou du génie de Rome; et il est à craindre que ce soit principalement à la France qu'ils adressent cette sorte de défi. Mais cette invention s'appuie sur un contresens historique qu'il n'est pas inutile peut-être de signaler... [l'auteur explique alors qu'Arminius, le Chérusque, appartenait à une nation qui fut plus tard à l'origine de la confédération des Francs, dont les Français sont bien évidemment les héritiers naturels]. Si nous avons préféré l'héritage de l'esprit romain à celui de l'esprit teutonique, nous ne pouvons cependant nous laisser dépouiller de nos ancêtres au point de souffrir patiemment que l'ennemi se serve de leurs noms contre nous.

Il y aurait ainsi toute une manière nouvelle pour nous de considérer l'histoire de la Germanie et les noms héroïques invoqués comme un patrimoine exclusif par la littérature allemande. Il n'y a pas jusqu'au poème épique des Niebelungen qui ne soit faussement revendiqué par les Allemands et qui ne puisse tout aussi bien être regardé comme la véritable épopée nationale des Francs ${ }^{11}$.

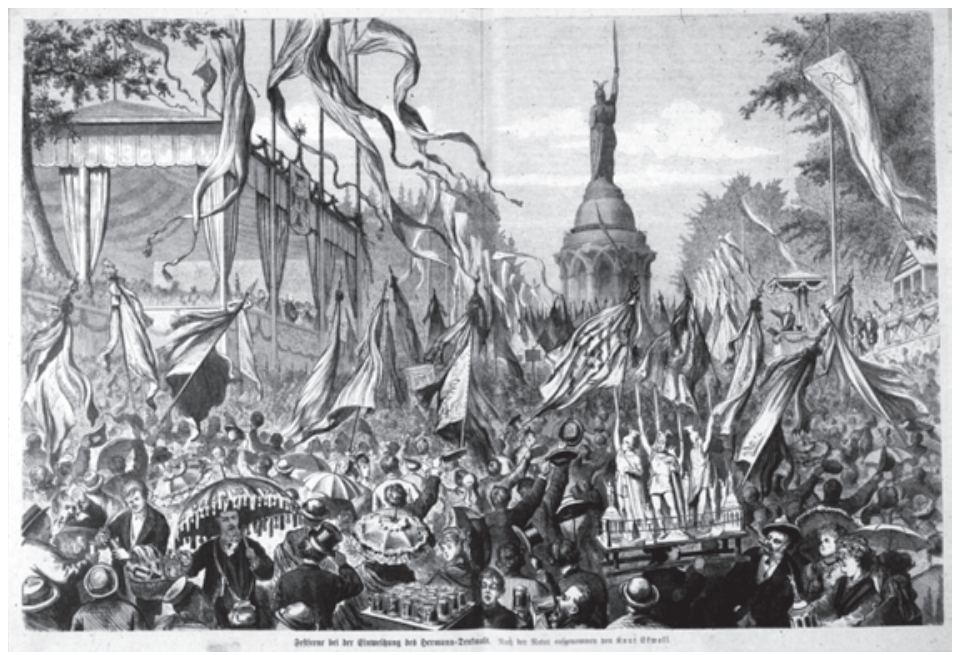

Fig. 2. L'érection de la statue d'Hermann

(Dessin de K. Ekwall paru dans Die Gartenlaube, Illustriertes Familienblatt, 1875.

D'après H. Buck, " Der Literarische Arminius-Inszenierungen einer sagenhaften Gestalt ", dans W. Schlüter (éd.), Kalkriese - Römer im Osnabrücker Land.

Archäologische Forschungen zur Varusschlacht, catalogue d'exposition, 1993, p. 280.)

11 Cité par Chr. Goudineau, Le dossier Vercingétorix (op. cit., note 9) p. 91. 
Bel exemple d'evocatio culturelle! Quel meilleur moyen pour dépouiller l'ennemi de son identité que de s'approprier sa mémoire nationale?

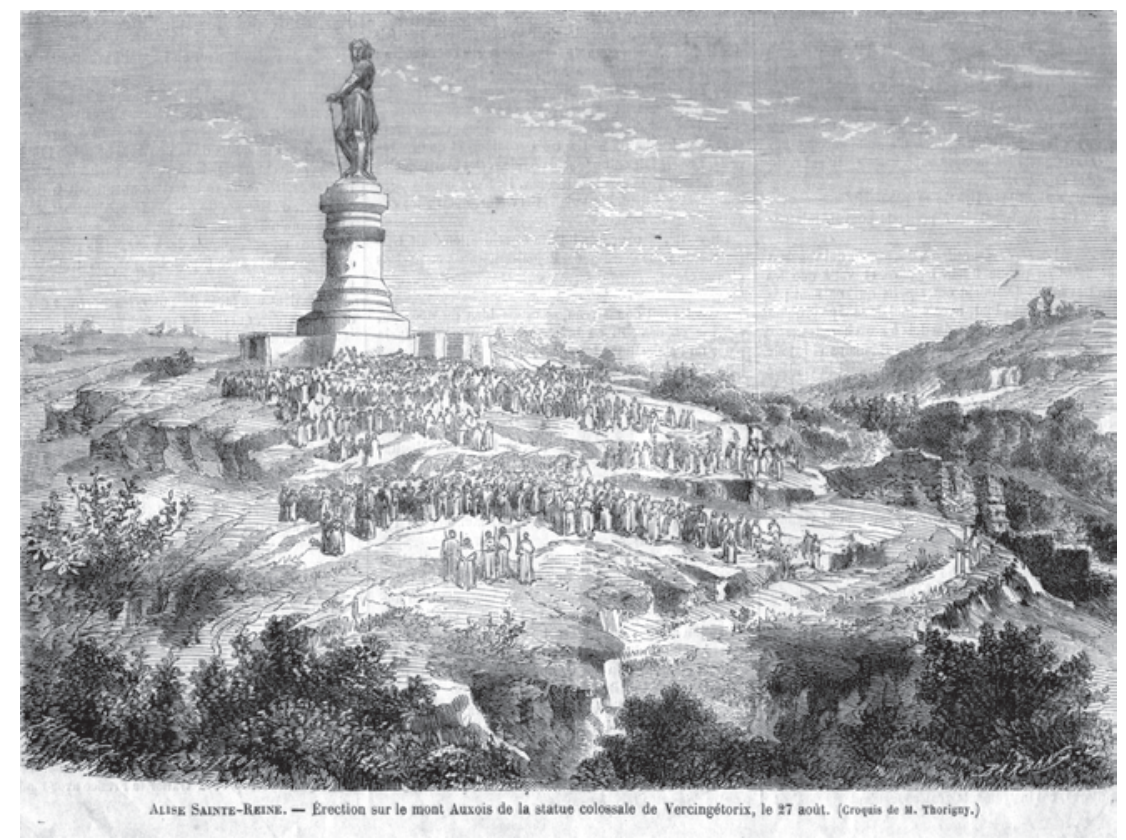

Fig. 3. L'érection de la statue de Vercingétorix sur le Mont-Auxois, le 27 août 1865 (Le Monde Illustré, 16 septembre 1865).

En attendant, l'Empire s'organise et son œuvre archéologique apparait considérable, d'une ambition scientifique sans commune mesure avec les tentatives pseudo-historiques qui l'ont précédé. Le monarque s'entoure d'un cercle de savants véritables dont l'influence est déterminante: Victor Duruy, Félix de Saulcy, Alexandre Bertrand, Léon Régnier, mais aussi Prosper Mérimée, William Fröhner, pour ne citer que ceux-là. En 1858 est créée la "Commission de topographie des Gaules " qui dispose d'un budget spécial pour mener des fouilles et des explorations. Celles d'Alésia seront notamment de sa responsabilité, avant d'être confiées à E. Stoffel. Suivront celles de Gergovie, de Mauchamp, du Puy d'Issolud, mais aussi les investigations dans la forêt de Compiègne, les premiers travaux sur le mont Beuvray, de nombreuses fouilles de tumulus protohistoriques dans l'Est de la France. L'intérêt de Napoléon III ne s'arrête pas à la France, il touche tout le bassin méditerranéen, comme l'a rappelé l'important colloque tenu à Compiègne en $2000^{12}$. L'empereur a notamment encouragé et financé les missions d'exploration de L. Heuzey en Macédoine, de G. Perrot en Asie Mineure, les fouilles d'E. Renan en Phénicie, celles dans Bulletin de la société historique de Compiègne 37 (2001), p. 15-303. 
de Roumanie, les missions épigraphiques de L. Renier en Algérie et en Italie. À Rome même, Napoléon III fait acheter les Orti Farnesiani, sur le Palatin, et confie la fouille du palais des Césars à Pietro Rosa. Les travaux dureront de 1861 à 1870, avant que le monarque déchu ne revende les terrains au tout jeune État italien ${ }^{13}$.

Mais cette entreprise a d'autres prolongements: on doit ainsi à l'Empereur l'acquisition de la collection Campana, brièvement exposée en 1862 dans l'éphémère musée Napoléon III, avec le fruit des missions conduites dans le bassin méditerranéen ${ }^{14}$; la même année parait le décret qui scelle la création du Musée de Saint-Germain-en-Laye, dont l'organisation est confiée à A. Bertrand. Son but est d'accueillir les antiquités de la France, depuis la plus lointaine préhistoire jusqu'à l'avènement du Christianisme en Gaule, selon un modèle inspiré de l'expérience du Musée des Antiquités du Nord, à Copenhague, et du Römisch-Germanisches Zentralmuseum de Mayence, créé par L. Lindenschmidt en 1852. Les échanges entre les deux institutions sont d'ailleurs fréquents, malgré les rivalités nationales ${ }^{15}$. Les moulages, comme à Mayence, y tiennent une place essentielle, car ils permettent de comparer, de confronter à distance les objets. C'est Napoléon III aussi qui fit exécuter ceux de la colonne Trajane à Rome, dont une copie fut donnée au Louvre avant d'être déposée à Saint-Germain ${ }^{16}$.

Tous ces faits sont bien connus et je ne les ai brièvement rappelés que pour remettre en perspective les fouilles entreprises sous l'égide de l'Empereur au pied du Mont-Auxois. Celles-ci ne sauraient en effet être isolées de leur contexte général. Bien qu'elles soient aujourd'hui les plus connues du grand public, il est important de se souvenir qu'elles s'inscrivent dans une œuvre beaucoup plus large, de nature véritablement scientifique, et qu'elles n'ont pas été inspirées, à fortiori dévoyées par la passion nationaliste. Certes elles participent d'une entreprise de "mémoire nationale " dans une Europe où s'aiguise l'affrontement des Nations modernes qui cherchent dans l'Histoire une justification de leur identité et de leurs prétentions. Mais, dans leur principe même et dans leur déroulement, elles servent une ambition scientifique estimable, avec bien sûr les moyens et les techniques de l'époque, qui nous font parfois sourire aujourd'hui ${ }^{17}$. Elles ne participent en rien d'une œuvre apologétique ou d'une

13 Voir supra, note 6.

14 È. Gran-Aymerich, "Le Palais de l'Industrie, miroir de la politique archéologique de Napoléon III", dans Napoléon III et l'archéologie (op. cit., note 12), p. 29-47.

15 F.W. von Hase, "Ludwig Lindenschmidt et Napoléon III. Un chapitre précoce de la coopération archéologique franco-allemande”, dans P. Jacquet et R. Périchon, Aspects de l'archéologie française au XIX siècle, Montbrison, 2000, p. 63-88.

16 A.-M. Liberati, "L'appropriazione dell'idea di Roma in chiave politica: Napoleone III, la Colonna Traiana e le collezioni del Museo della Civiltà Romana", dans Napoléon III et l'archéologie (op. cit., note 12), p. 291-303.

17 J'ai rappelé ailleurs les principes techniques qui ont guidé ces travaux et qui, pour l'époque, sont réellement sérieux et novateurs. Voir M. Reddé, "Les fouilles du second Empire autour d'Alésia, à la lumière des recherches récentes”, dans Napoléon III et l'archéologie (op. cit., note 12), p. 93-115. 
entreprise cocardière. Il s'agissait de se donner les moyens d'observer sur le terrain, par une démarche expérimentale nouvelle, les indices matériels qui permettaient de vérifier une théorie que les textes seuls ne pouvaient totalement corroborer. Et, je l'ai rappelé, elles ne sont pas le point de départ, mais au contraire le point final d'une querelle sur la localisation d'Alésia, commencée bien avant le premier coup de pioche, même si cette querelle a rebondi beaucoup plus tard.

Et puis il y eut Sedan. La France vaincue, humiliée, dépouillée, assoiffée de revanche. Alésia devient l'archétype de la défaite et Vercingétorix le héros douloureux qui se sacrifie pour la patrie, dans un geste plein de noblesse et de fierté, après avoir combattu jusqu'au bout. Ce n'est pas là une interprétation moderne, mais l'opinion directement exprimée par les témoignages de l'époque, quelle qu'en soit l'origine sociale ou intellectuelle. Commençons par ce texte de F. Coppée, écrit en 1880:

Dans les brumeuses origines de la France, aucune figure n'apparaît plus grande que celle de Vercingétorix; elle domine notre histoire nationale comme la belle statue d'Aimé Millet, coiffée du casque ailé et s'appuyant sur sa lourde hache, domine de sa taille colossale les vertes vallées de la Côte-d'Or, du haut du Mont-Auxois. C'est une noble pensée que d'avoir élevé ce monument au glorieux vaincu; et nos tristesses patriotiques de l'heure présente songent, avec un mélancolique orgueil, à l'indomptable courage du chef barbare et à sa lutte opiniâtre contre l'invasion romaine. Ceux qui avaient l'âge de raison en 1870 nous comprendront. Ils ont senti comme nous, à la seule nouvelle que l'ennemi avait franchi la frontière, l'horrible frisson de la vierge violée; ils ont éprouvé l'instinctif besoin de vengeance, fait le geste machinal de saisir une arme, appelé furieusement la victoire ou la mort; et après avoir vu la gorge ouverte de la patrie râler sous la botte du Prussien, ils ont gardé au fond du cœur une rancune éternelle.

Voici maintenant, en contrepoint, un bref passage extrait du Tour de France par deux enfants, histoire populaire publiée en 1877 qui connut un vif succès. Le récit met en scène deux petits orphelins partis de Phalsbourg à la porte de l'Alsace. Julien, le plus âgé, lit à Jean-Joseph, son cadet, une histoire de France qui commence par la conquête romaine:

Un jeune Gaulois, né dans l'Auvergne, résolut alors de chasser les Romains du sol de la patrie. Il parla si éloquemment de son projet à ses compagnons que tous jurèrent de mourir plutôt que de subir le joug romain. En même temps, ils mirent à leur tête le jeune guerrier et lui donnèrent le titre de Vercingétorix, qui veut dire chef.

Bientôt Vercingétorix envoya en secret dans toutes les parties de la Gaule des hommes chargés d'exciter les Gaulois à se soulever. On se réunissait la nuit sous l'ombre impénétrable des grandes forêts, auprès des énormes pierres qui servaient d'autels; on parlait de la liberté, on parlait de la patrie, et l'on promettait de donner sa vie pour elle...

Au jour désigné d'avance, la Gaule entière se souleva d'un seul coup, et ce fut un réveil si terrible que, sur plusieurs points, les légions romaines furent exterminées. 
César, qui se préparait alors à quitter la Gaule, fut forcé de revenir en toute hâte pour combattre Vercingétorix et les Gaulois révoltés. Mais Vercingétorix vainquit César à Gergovie.

- Gergovie, dit Jean-Joseph, c'est un endroit à côté de Clermont, j'en ai entendu parler plus d'une fois. Continuez, Julien; j’aime ce Vercingétorix.

Six mois durant, Vercingétorix tint tête à César, tantôt vainqueur, tantôt vaincu.

Enfin César réussit à enfermer Vercingétorix dans la ville d'Alésia, où celui-ci s'était retiré avec soixante mille hommes.

Alésia, assiégée et cernée par les Romains, comme notre grand Paris l'a été de nos jours par les Prussiens, ne tarda pas à ressentir les horreurs de la famine.

- Oh! dit Julien, un siège, je sais ce que c'est: c'est comme à Phalsbourg, où je suis né et où j'étais quand les Allemands l'ont investi. J'ai vu les boulets mettre le feu aux maisons, Jean-Joseph; papa, qui était charpentier et pompier, a été blessé à la jambe en éteignant un incendie et en sauvant un enfant qui serait mort dans le feu sans lui...

L'assimilation de la défaite d'Alésia à celle de Sedan n'est pas alors perçue d'une manière différente en Allemagne. Th. Mommsen n'hésite pas en effet à tirer de la victoire de César des leçons valables pour le présent:

Le hasard ne fit pas cette grande catastrophe. Si parfois il la prépare pour les peuples susceptibles d'une haute culture, ici, il faut le dire, les Gaulois ne tombèrent que par leur propre faute. Leur ruine était en quelque sorte historiquement nécessaire: toute cette dernière guerre le prouve, qu'on en étudie la marche, soit dans l'ensemble, soit dans les détails. À l'heure où menaçait la domination étrangère, il ne se rencontra de résistance énergique que chez quelques clans isolés, et ceux-ci même, Germains pour la plupart ou à demi Germains... Tels se sont montrés tous les Gaulois au siècle de César: ni puissante organisation militaire, ni discipline politique: ils ne purent y atteindre, ils ne les auraient pas supportées! Dans tous les temps, dans tous les lieux, vous les voyez toujours les mêmes, faits de poésie et de sable mouvant, à la tête faible, au sentiment profond, avides de nouveautés et crédules, aimables et intelligents, mais dépourvus du génie politique: leurs destinées n'ont pas varié: telles elles furent autrefois, telles elles sont de nos jours ${ }^{18}$.

Innombrables sont les textes que l'on pourrait citer et qui montrent comment la III ${ }^{e}$ République a instrumentalisé Vercingétorix, premier héros national, et Alésia, haut lieu de la mémoire douloureuse de la France ${ }^{19}$. Dans cette re-construction identitaire, les manuels scolaires du temps ont joué un rôle considérable, mais il faudrait naturellement une analyse plus fine pour montrer la différence sensible qui existe, sur ce point,

18 Th. Mommsen, Histoire romaine, Livre V, chap. VII, éd. "Bouquins ", Paris, Laffont, 1985, p. 213.

19 Chr. Amalvi, De l'art et la manière d'accommoder les héros de l'histoire de France. Essais de mythologie nationale, Paris, 1998. 
entre l'enseignement public et l'enseignement catholique ${ }^{20}$. Tel n'est pas ici mon propos. Il n'est pas sans intérêt, en revanche, de rappeler que cette utilisation du mythe historique trouve son parallèle à la même époque dans les manuels scolaires allemands où Arminius et la bataille du Teutoburg occupent une place sensiblement identique à celle de Vercingétorix et d'Alésia ${ }^{21}$.

Cette manière d'accommoder "Nos ancêtres les Gaulois » touche tous les domaines du savoir et de la culture, comme l'a bien montré l'important colloque tenu en 1982 à Clermont-Ferrand, et auquel je me contente de renvoyer, faute de pouvoir l'analyser en détail ${ }^{22}$. Je citerai seulement ici, pour le plaisir de l'anecdote, le sort de ces deux statues de Vercingétorix, érigées l'une à Bordeaux, l'autre à Saint-Denis, puis enlevées et fondues par les troupes allemandes pendant la Seconde Guerre mondiale, signe indubitable que les enjeux identitaires étaient bien perçus de manière parallèle par les deux adversaires ${ }^{23}$. Celle de A. Bartholdi, à Clermond-Ferrand, échappa à ce sort: on était sans doute trop près de Vichy. Celle du Mont-Auxois, qui faillit aussi y passer, était devenue " lieu de mémoire " avant la Première Guerre mondiale. On $y$ venait en pèlerinage, le beau monde s'y pressait, hommes politiques, universitaires et savants, artistes, prélats même, comme Monseigneur Baudrillard. On y lisait des discours patriotiques, on y jouait des drames, on prononçait des vers, on invitait les enfants des écoles, pour une leçon d'instruction civique. Et bien sûr la journée se terminait par un banquet... On ne faisait plus guère à ce moment, dans le registre savant, et ce n'était plus vraiment le moment d'une querelle sur l'identification du lieu de la célèbre bataille, la question était résolue pour tout le monde, hormis quelques hurluberlus ${ }^{24}$. On faisait plutôt dans l'outrance verbale, tel Jules Toutain, qui reprenait avant guerre les fouilles sur l'oppidum et dressait peu après un parallèle injurieux au titre évocateur (Héros et bandit. Vercingétorix et Arminius, Paris, 1916) : "La comparaison se justifie aisément. Tous deux ont voulu délivrer leur patrie du joug romain. [Mais] Vercingétorix ne cessa pas de mener la lutte en soldat; [...] à aucun moment de son récit César ne fait le moindre reproche à Vercingétorix ni à ses troupes [...] les deux adversaires combattent, pour ainsi dire, à visage découvert. Il ne s'y trouve pas un seul épisode qui évoque des idées de fourberie, de bassesse, de déloyauté. Bien plus,

Chr. Amalvi, "La construction du mythe de la bataille d'Alésia dans la littérature populaire et scolaire du Second Empire à la Cinquième République", dans M. Reddé et S. von Schnurbein (ed.), Alésia et la bataille du Teutoburg... (op. cit., note 8), p. 121-132. R. Riemenschneider, "Le mythe national de la bataille du Teutoburg dans les manuels scolaires allemands", dans M. Reddé et S. von Schnurbein (ed.), Alésia et la bataille du Teutoburg... (op. cit., note 8), p. 133-151.

P. Viallaneix et J. Ehrard, Nos ancêtres les Gaulois, Faculté des Lettres et Sciences humaines de l'Université de Clermond-Ferrand II, fasc. 13, 1982.

24 M. Reddé, "La querelle d'Alésia, hier et aujourd'hui”, dans M. Reddé et S. von Schnurbein (éd.), Alésia et la bataille du Teutoburg... (op. cit., note 8), p. 155. 
l'auteur [...] insiste à plusieurs reprises sur l'héroïsme et la grandeur d'âme des Gaulois et de leur chef: il rend hommage aux éminentes qualités de Vercingétorix. " Arminius, par contre, c'est « la cruauté naturelle des Germains [...] la traîtrise et la perfidie dans la préparation, la fourberie et la lâcheté dans l'exécution, la férocité raffinée et la bassesse dans le traitement infligé aux vaincus ». Il est vrai qu'on était en guerre!

On devrait sans doute se demander pourquoi ce n'est pas Gergovie, symbole de victoire, mais Alésia, symbole de défaite, qui est devenue à cette époque le lieu de mémoire de la France. Sans doute l'assimilation avec le désastre de Sedan a-t-elle joué à plein dans la conscience collective de la III République. C’est le régime de Vichy, au contraire, qui a tenté de redonner du lustre à Gergovie, haut lieu de la gloire française, peut-être simplement pour des raisons pratiques: on était en zone libre, Alise en zone occupée, et il n'était pas question de venir y manifester. Le mythe gaulois fut une nouvelle fois mobilisé par la " Révolution nationale " dans la bataille idéologique de ces temps troublés, ce qui donna lieu à une invraisemblable cérémonie que l'on hésite à qualifier: le 30 août 1942, en présence d'une foule nombreuse et de milliers de membres de la Légion, le Maréchal Pétain déposait à Gergovie, dans une crypte-reliquaire, une parcelle de terre de chaque commune, « recueillie dans la métropole et dans les colonies, sur tous les lieux où souffle l'esprit de la France et où se garde le souvenir de ceux qui en ont fait la grandeur ${ }^{25} » \ldots$

L'effondrement du régime allait refaire d'Alésia un symbole national, après guerre. Mais alors, bien entendu, c'est la " résistance » à l'éternelle menace germanique et l'union avec la civilisation romaine qui reprit le dessus. Sur le mur de la gare des Laumes, vous pouvez toujours voir la plaque qui porte cette inscription: «1949. Dans cette plaine, il y a deux mille ans, la Gaule a sauvé l'honneur en opposant à la voix de Vercingétorix ses peuples aux légions de César. Après l'échec de ses armes, réconciliée avec le vainqueur, unie, défendue contre les invasions germaniques, ouverte aux lumières de la Grèce et de Rome, elle a connu trois siècles de paix. »

Nos ancêtres les Gaulois, Vercingétorix, Alésia: le mythe aujourd'hui a du plomb dans l'aile, et c'est tant mieux. Ses avatars montrent comment l'archéologie a été instrumentalisée au profit d'une histoire immédiate inscrite dans la trame des conflits qui ont secoué l'Europe au $\mathrm{XIX}^{\mathrm{e}}$ et au $\mathrm{XX}^{\mathrm{e}}$ siècle, au bénéfice d'une quête identitaire dont les préoccupations n'étaient guère scientifiques. De ce point de vue, la période du Second Empire fait figure d'heureuse exception, avec une politique archéologique d'une tout autre ambition intellectuelle.

25 K. Pomian, "Francs et Gaulois", dans P. Nora (éd). Les lieux de mémoire. III. Les France, Paris, 1992, p. 41-105. 
"Quels titres a donc l'Allemagne à continuer Rome? Pourquoi ce rôle ne reviendrait-il pas à la France...? » écrivait C. Jullian ${ }^{26}$. On me demande souvent pourquoi j’ai invité une équipe allemande à participer aux nouvelles fouilles d'Alésia, ce haut lieu de la mémoire nationale française: parce qu'il était temps d'en finir avec cette histoire-là, justement.

Michel REDDÉ

INHA / École Pratique des Hautes Études

2, rue Vivienne

F-75002 Paris

redde.michel@yahoo.fr

26 C. Jullian, Au seuil de notre histoire. Leçons faites au Collège de France, II (1914-1923), Paris, 1931, X, p. 24. 